Revista Española de Antropología Americana ISSN: 0556-6533

https://doi.org/10.5209/reaa.64964

\title{
Un conjunto arquitectónico asociado con la elite de Sihó, Yucatán
}

\author{
Rafael Cobos ${ }^{1}$ y Alfonso Lacadena García-Gallo ${ }^{2} \uparrow$
}

Recibido: 23 de febrero de 2019 / Aceptado: 28 de marzo de 2019

Resumen. Trabajos de investigación arqueológica realizados en Sihó, Yucatán, a principios del siglo XXI, revelaron la existencia de un conjunto arquitectónico asociado con individuos pertenecientes a la elite. Este grupo de habitación se encuentra a corta distancia del centro del asentamiento y proporciona información sobre la disposición espacial de elaborados grupos arquitectónicos asociados con unidades residenciales en un tipo de asentamiento maya del periodo Clásico Tardío. En esta investigación se emplean restos arquitectónicos, materiales suntuarios y evidencia de escritura en un panel para argumentar que los ocupantes del conjunto arquitectónico fueron individuos privilegiados y gozaron de opulencia.

Palabras clave: élite; asentamiento; unidad doméstica; Ajaw.

\section{[en] An Architectural Compound Associated with the Elite at Sihó, Yucatán}

\begin{abstract}
Early XXI century archaeological research conducted at Sihó, Yucatán, revealed the existence of an architectural compound associated with elite members. This compound is located at a short distance to the northwest of the site-center. It provides information on the spatial distribution of elaborate architectural groups linked with residential units in one type of Maya settlements dating back to the Late Classic period. Architectural remains, vestiges of sumptuary objects, and evidence of a carved panel suggest that the compound's residents were privileged individuals living an opulent lifestyle.
\end{abstract}

Keywords: Elite; settlement; domestic unit; Ajaw.

Sumario. 1. Introducción. 2. Sihó: una comunidad prehispánica del Clásico Tardío. 3. Materiales asociados a las estructuras investigadas. 4. Conclusiones. 5. Referencias.

Cómo citar: Cobos, Rafael y Alfonso Lacadena García-Gallo. 2019. «Un conjunto arquitectónico asociado con la elite de Sihó, Yucatán». Revista Española de Antropología Americana 49 (número especial): $139-155$.

\section{Introducción}

Los estudios de la organización espacial interna de las comunidades mayas prehispánicas, continúa siendo un elemento importante y determinante en el análisis e identificación de los miembros que integraron esas comunidades del pasado. Los restos materiales del quehacer cotidiano realizado por personas, ya sea de manera

\footnotetext{
1 Facultad de Ciencias Antropológicas, Universidad Autónoma de Yucatán.rachcobos@aol.com

2 Departamento de Historia de América y Medieval y Ciencias Historiográficas. Universidad Complutense de Madrid.
} 
individual y/o colectiva, quedaron plasmados físicamente en el asentamiento, cuyos componentes básicos o esenciales incluyen: a) una parte central, b) una parte homogénea, c) áreas que permitieron la circulación, d) partes especiales (Doxiadis 1968).

Numerosos investigadores, estudiando patrones de asentamiento en sitios mayas de las Tierras Bajas, han identificado los cuatro componentes básicos a los que se refiere Doxiadis (1968). Estos estudiosos los han asociado con distintas formas de comunidades prehispánicas y su componente social. Por ejemplo, los asentamientos mayas de las Tierras Bajas se han relacionado con áreas ocupadas tanto por individuos privilegiados, poderosos y con opulencia, como por gentes pertenecientes a sectores menos privilegiados, es decir, la gran población o individuos campesinos y/o artesanos, quienes carecieron de sobreabundancia (ver por ejemplo, Haviland 1965; Kurjack 1974; Kurjack y Garza 1981; Fash 1983; Folan et al. 1983; Gallareta 1984; Tourtellot 1988; Chase y Chase 1992; Haviland y Moholy-Nagy 1992; Tourtellot et al. 1992; Chase 1998; Folan et al. 2001; Cobos 2003; Barnhart 2006).

Los cuatro componentes básicos definidos por Doxiadis (1968) pueden emplearse para interpretar que la distribución espacial de personas pertenecientes a la elite y no-elite ocurrió de dos formas particulares en los asentamientos mayas. En la primera, reconocemos que miembros de la elite se asentaron en la parte central del asentamiento mientras que el grueso de la población integrado por individuos que no eran de la elite ocupó la parte homogénea que rodeaba el centro. En la segunda forma, individuos de la elite se establecieron tanto en la parte central como en la parte homogénea que rodeaba el centro del asentamiento. Esta distribución espacial propició que se mezclaran miembros de la elite y no-elite en el amplio paisaje del asentamiento localizado más allá de la parte central. Además, estas dos formas espaciales de los asentamientos mayas prehispánicos fueron contemporáneas y una no excluyó a la otra.

En el primer caso mencionado, la parte central del asentamiento con elaboradas construcciones arquitectónicas y edificios abovedados fue ocupada por miembros de la elite y, en la periferia del área central, habitó la gran población integrada por individuos quienes no eran de la elite. Esta particular distribución espacial del asentamiento maya fue descrita por Diego de Landa (1959) en el siglo XVI cuando se refirió a comunidades prehispánicas que habían adquirido su forma definitiva al final del periodo Posclásico y en vísperas del contacto con los españoles. Datos arqueológicos del asentamiento de Dzibilchaltún fechados para el periodo Clásico Tardío (600-800) también corroboran esta forma de arreglo espacial (Kurjack 1974).

También han sido ampliamente documentados en sitios como Calakmul, Cobá, Caracol, Chichén Itzá, Ceibal, Copán, Palenque (Fash 1983; Folan et al. 1983; Gallareta 1984; Tourtellot 1988; Chase 1998; Folan et al. 2001; Cobos 2003; Barnhart 2006), ejemplos asociados al segundo caso, con individuos de la elite residiendo tanto en la parte central como periferia de la comunidad, así como personas no pertenecientes a la elite asentadas en la parte homogénea del asentamiento. La residencia y convivencia de individuos pertenecientes tanto a la elite como aquellos que no pertenecían a ella en el área homogénea puede explicarse por caminos o calzadas artificialmente construidas que permitieron la circulación, es decir, el tercer componente definido por Doxiadis (1968). La expresión material de esta circulación revela dos aspectos espaciales. Primero, las antiguas calzadas mayas conectaron o relacionaron la periferia con el centro. Segundo, numerosos grupos arquitectónicos de la élite asentada $\mathrm{u}$ ocupando la periferia también se relacionaron entre sí al haber utilizado 
calzadas que nunca pasaron por el área central del asentamiento, como se ha documentado en Chichén Itzá y Caracol (Chase 1998; Cobos 2003).

Considerando las dos formas de arreglo espacial de los asentamientos mayas mencionadas líneas arriba, en este trabajo fijamos nuestra atención solamente en una de ellas. De manera particular nos referimos a la distribución espacial que involucra el área central, con su imponente y compleja arquitectura elaborada de mampostería, y la periferia que carece o no tiene las complejas y elaboradas edificaciones. Este tipo de asentamientos contienen numerosas unidades domésticas que, conforme uno se aleja del centro, dejan de exhibir la elaborada arquitectura de mampostería con edificios techados con bóvedas.

A mediados de la década de 1950, Willey (1956: fig. 1) empleó un esquema idealizado -en vez de datos arqueológicos- para proponer tres tipos de asentamientos mayas a los que denominó A, B y C. Cabe indicar que esta idealización fue resultado de la carencia de datos arqueológicos directamente relacionados con las antiguas comunidades mayas prehispánicas, ya que este tipo de estudios no se inició de manera sistemática hasta la década de 1960 en Tikal y Dzibilchaltún (Haviland 1965; Kurjack 1974). De los tres tipos de asentamiento, nos interesa el «Asentamiento Tipo $\mathrm{B} \gg$ que Willey (1956: 111) caracterizó por poseer unidades residenciales separadas a distancias que oscilan entre 400 y $800 \mathrm{~m}$ y distribuidas en el terreno que constituye la periferia del centro. El espacio que separa las unidades domésticas pudo haber sido utilizado, por ejemplo, como áreas verdes para practicar agricultura extensiva y/o arboricultura, silvicultura y horticultura (Harrison y Turner 1978; Graham 1999), o bien, pudo haberse dejado sin tocar o cultivar para permitir crecer la vegetación y así crear barreras naturales entre las unidades domésticas (Kurjack 1974: 74).

En este trabajo nos enfocamos en una unidad doméstica -Tipo B- ubicada aproximadamente $200 \mathrm{~m}$ al noroeste del centro del asentamiento de Sihó (Yucatán). Los restos materiales sugieren que se trató de un conjunto residencial asociado con la elite ya que reconocemos lo siguiente: evidencia material de haberse invertido numerosas horas-hombre en la construcción del conjunto residencial que incluye un gran basamento con escalinata; un edificio abovedado con dos crujías; dos construcciones sencillas cuyas paredes y techumbre fueron de materiales perecederos y funcionaron como construcciones auxiliares; una cisterna o chultún para el almacenamiento de agua; banco de material para extraer material de caliza para la construcción; obtención y uso de materiales elaborados en cerámica, sílex y obsidiana que pueden considerarse como bienes suntuarios o de lujo y un panel de caliza tallado con la fecha 12 Ajaw.

Los restos materiales asociados con la unidad doméstica de Sihó se utilizan para contestar tres preguntas. Primero, ¿qué tipo de actividades realizaron cotidianamente los residentes de la unidad doméstica? Segundo, ¿quiénes residieron en esa elaborada unidad doméstica? Tercero, ¿qué relación pudieron haber tenido con miembros de la elite quienes ocuparon la parte central de Sihó?

\section{Sihó: una comunidad prehispánica del Clásico Tardío}

La antigua comunidad prehispánica de Sihó se encuentra en la parte occidental de Yucatán (Figura 1). El sitio está registrado en el Atlas Arqueológico del Estado de Yucatán como un asentamiento de tercer rango y sus coordenadas geográficas son 


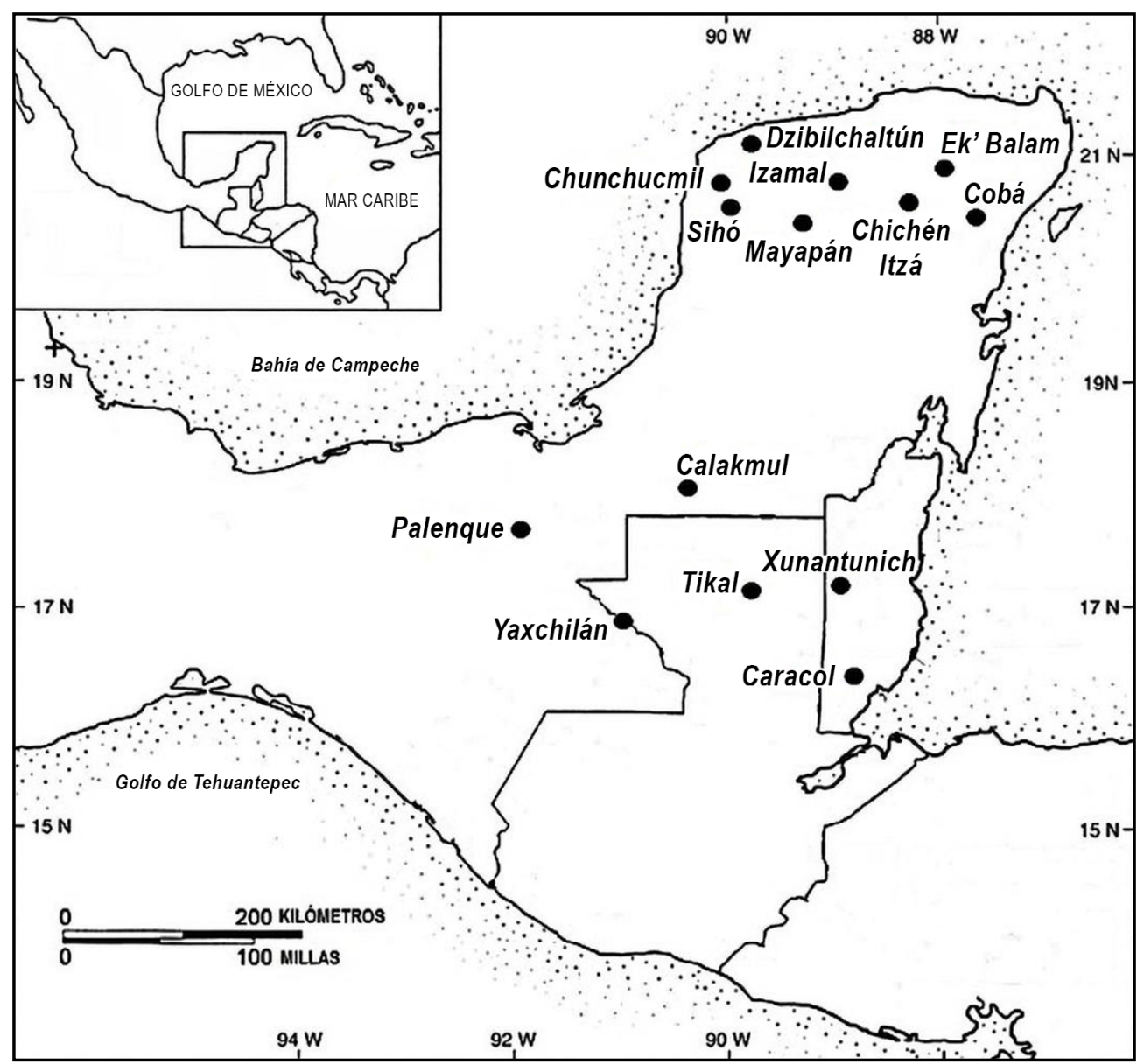

Figura 1. Ubicación de Sihó en el occidente de Yucatán (dibujo de Rafael Cobos).

15Q YN950681 (Garza y Kurjack 1980: 88). De acuerdo a la definición de Garza y Kurjack (1980: 32; ver también Willey 1956), los sitios de tercer rango se caracterizan por presentar un grupo central con arquitectura monumental, conjuntos arquitectónicos alrededor de este grupo central y por estar estos unidos por una o más calzadas. La distribución espacial del asentamiento de Sihó coincide en gran medida con esta definición, e incluso existe una única calzada en el centro del asentamiento que conecta dos grupos arquitectónicos.

La unidad doméstica a la que nos referimos en este trabajo está integrada por tres construcciones que incluyen las estructuras 5D16, 5D19 y 5D20 que se asientan o descansan sobre la superficie de un gran basamento (Figura 2). De estas tres construcciones, 5D16 es el edificio más importante e imponente ya que es una construcción elaborada con muros de mampostería y techo de bóvedas escalonadas. En lo referente a 5D19 y 5D20, estas dos estructuras exhiben cimientos de piedra de forma rectangular que sostuvieron materiales perecederos tanto en sus muros como la techumbre (Fernández et al. 2003a, 2003b; Tun Ayora 2004; Fernández 2008). 
Figura 2. Localización de la unidad doméstica 5D16, 5D19 y 5D20 en relación con el centro de Sihó al suroeste.

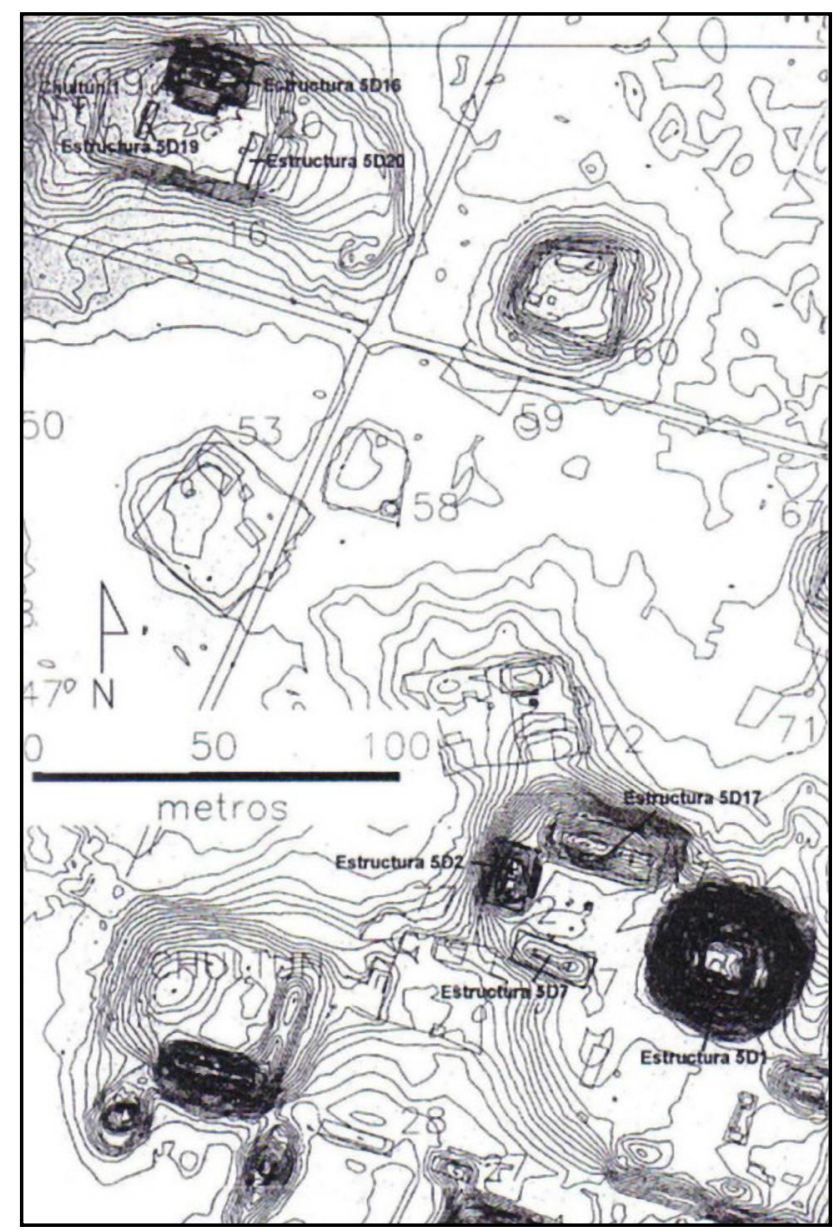

El arreglo espacial de 5D16, 5D19 y 5D20 muestra que la primera de ellas se construyó en el lado norte del basamento, en tanto que 5D19 y 5D20 se edificaron al oeste y este respectivamente, las tres estructuras miran hacia un pequeño espacio abierto, o patio central (Figura 3), libre de rasgos arqueológicos y cuya excavación no reveló ni basureros ni tampoco otras concentraciones significativas de materiales culturales. Por su ubicación espacial como centro de las mencionadas edificaciones esta plaza central debió de haber permitido lo siguiente: a) la circulación de individuos que ocupaban las tres construcciones, b) el movimiento de personas que ascendieron desde el lado sur a la unidad doméstica por la gran escalinata hecha con bloques de piedra megalíticos, y c) el encuentro entre individuos y la reunión de personas, promoviendo con esto interacciones sociales entre miembros de la comunidad y entre estos y la autoridad central.

\subsection{Estructura 5D16}

La 5D16 es una estructura tipo palacio ya que posee crujías alargadas, lo que la relaciona con un edificio de función habitacional asociado con individuos pertene- 


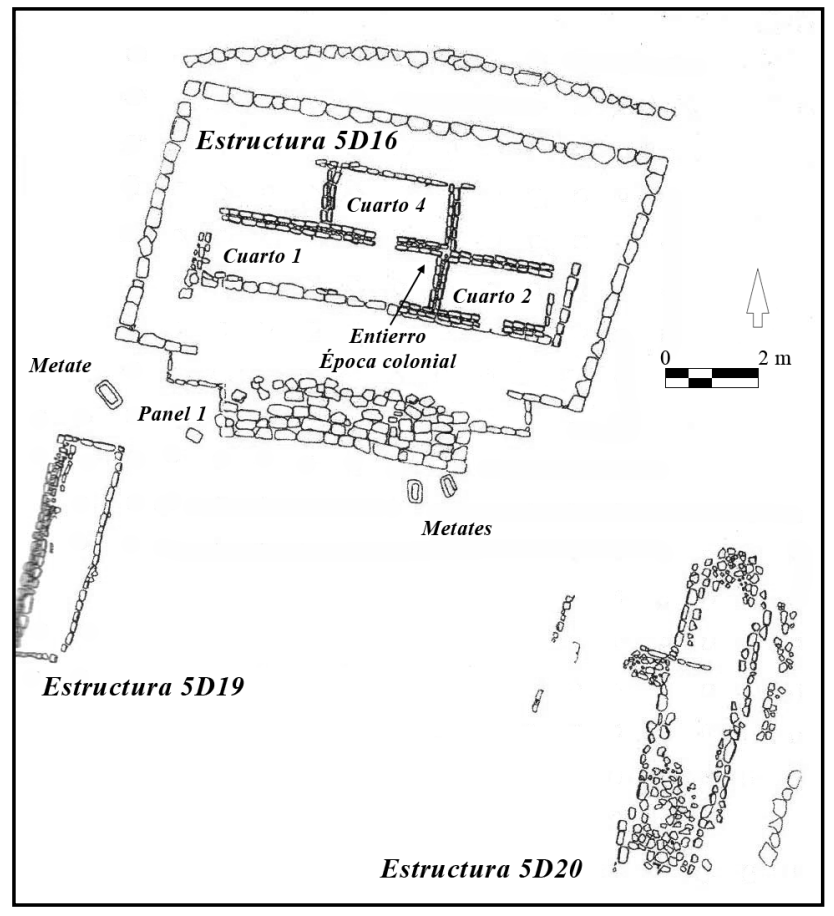

Figura 3. Unidad doméstica formada por 5D16, 5D19 y 5D20 (dibujo de Rafael Cobos).

cientes a la elite. Además, cuando consideramos el volumen métrico en su diseño y elaboración arquitectónica, las construcciones tipo palacio son la huella material de numerosas horas-hombre invertidas en su construcción y acabado final, ya que requirieron tanto de una gran cantidad de material en su edificación como de una compleja organización laboral (Kurjack 1994; Hoil Chacón 2006).

El basamento sobre el que descansa la Estructura 5D16 mide 16,5 m de ancho por $21 \mathrm{~m}$ de largo, incluyendo la escalinata, y está construido con piedras megalíticas (Figuras 4 y 5). En la parte superior del edificio se sitúan dos crujías de $16 \mathrm{~m}$ de longitud, que a su vez están divididas por muros que fueron agregados para delimitar los cuartos de esta parte superior de la estructura (ver Figura 3). Los cuartos 1 y 2 se localizan en el lado sur de 5D16, en tanto que los cuartos 3, 4 y 5 están dispuestos en un eje este-oeste en la parte norte de la construcción. Los cuartos 1, 2 y 4 son los mejor conservados de la construcción ya que claramente se distinguen sus muros de mampostería.

El Cuarto 1 de 5D16 es el más amplio del edificio y presenta un único acceso en su lado sur, es decir, hacia la escalinata y plaza central del conjunto. En algún momento de la historia del edificio, y aparentemente cuando ya no funcionaba como una estructura tipo palacio asociada con la elite, al acceso principal del Cuarto 1 se le colocó un alineamiento de piedras irregulares de baja altura (menos de $50 \mathrm{~cm}$ ). Este alineamiento no fue obstáculo para continuar ingresando al interior del edificio $\mathrm{y}$, como prueba, nos referimos al entierro infantil depositado en algún momento de la época Colonial en la esquina noreste del interior del Cuarto 1 (Fernández et al. 2010). El alineamiento de piedras irregulares podría haber sido contemporáneo a la colocación de la urna y este acto se realizó en algún momento de la época Colonial (Figura 6). Además, la colocación de las piedras sirvió para delimitar un espacio funerario que individuos del periodo Colonial le asignaron a 5D16. Es muy probable 
Figura 4. Vista sur-norte del lado este de la Estructura 5D16 (el Cuarto 2 aparece en segundo plano; fotografía de Rafael Cobos).
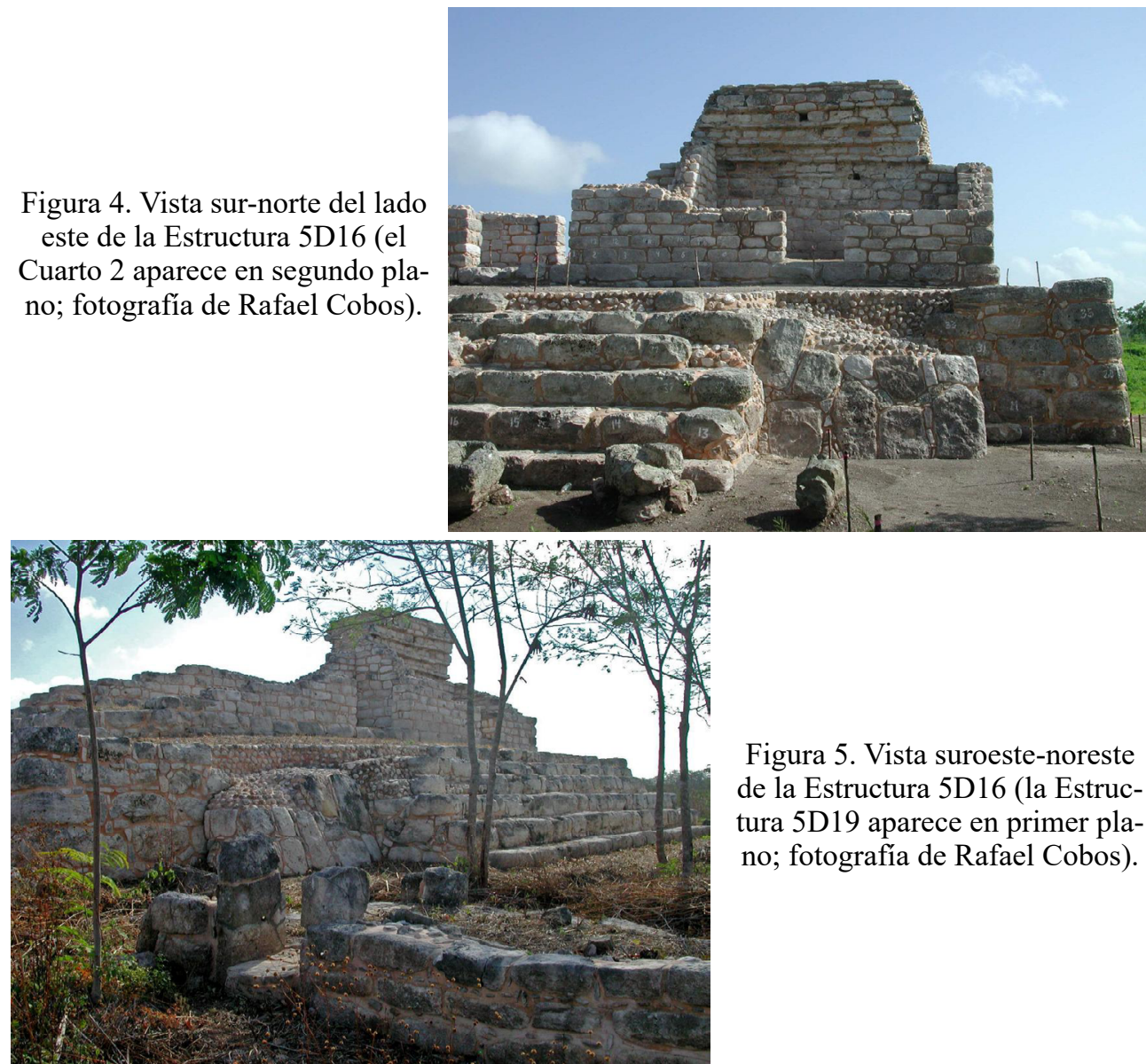

Figura 5. Vista suroeste-noreste de la Estructura 5D16 (la Estructura 5D19 aparece en primer plano; fotografía de Rafael Cobos).

Figura 6. Detalle de urna hallada en el Cuarto 1, Estructura 5D16 (fotografía de Rafael Cobos).

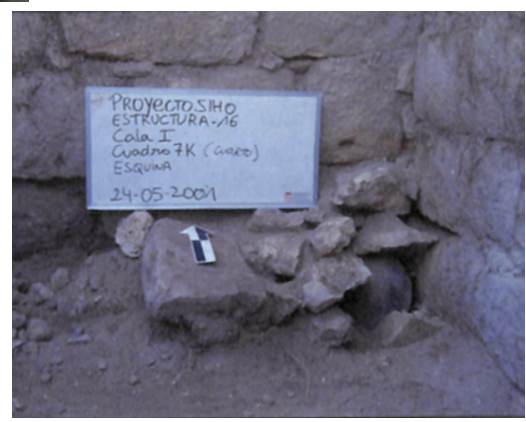

que no haya ningún tipo de vinculación entre estos individuos y aquellos que ocuparon el edificio unos 800 años antes.

Al norte del Cuarto 1, un pequeño acceso permite el ingreso al Cuarto 4. El Cuarto 2 fue construido al lado este del Cuarto 1 y posee su propio acceso, que también mira hacia la escalinata y plaza del conjunto. Cabe apuntar que las excavaciones del edificio no revelaron un acceso que permitiera la comunicación entre los cuartos 1 y 2. Hacia el lado norte de 5D16 se distinguen cuatro muros que definen el Cuarto 4 justo al centro de la construcción. Los cuartos 3 y 5 flanquearon hacia el oeste y 
este respectivamente el Cuarto 4 pero, debido a su mal estado de conservación, fue imposible determinar la ubicación de sus correspondientes accesos.

\subsection{Estructura 5D19}

En cuanto a la Estructura 5D19, esta construcción es de planta rectangular orientada en un eje norte-sur, tiene una longitud que oscila entre 9 y 9,70 metros y una anchura que varía entre 1,90 y 2,10 metros (ver Figura 3). 5D19 estuvo techada con materiales perecederos y el área de cobertura fue de 19,16 $\mathrm{m}^{2}$. El lado oeste de 5D19 está formado por un muro doble de aproximadamente $60 \mathrm{~cm}$ de ancho, en tanto que, un alineamiento de piedras rectangulares, delimitan los lados norte, sur y este de la estructura. La altura del muro oeste fue -aparentemente- de $92 \mathrm{~cm}$ y, para su construcción, se emplearon piedras irregulares y reutilizaron piedras de cornisa y un fragmento de panel esculpido (Tun Ayora 2004; Fernández 2008). Los lados norte y sur de la estructura pudieron haber estado abiertos, o bien, sus paredes pudieron haber estado realizadas por materiales perecederos. El lado este de 5D19 se mantuvo abierto para permitir el ingreso desde la plaza central al interior de la construcción.

En la parte noroeste de 5D19 se encuentra un acceso que se distingue por la presencia de dos jambas que delimitan un espacio abierto de $66 \mathrm{~cm}$. Este acceso comunica el interior de la estructura con un espacio exterior abierto y la cisterna o chultún (Tun Ayora 2004; Fernández 2008).

\subsection{Estructura 5D20}

La 5D20 es una construcción simple hecha de piedras irregulares grandes y medianas. Esta estructura tiene una longitud de $15 \mathrm{~m}$ y ancho de entre 3,80 (al norte) y 4,00 $\mathrm{m}$ (al sur); se estima que el área interna techada de 5D20 pudo haber sido de 35,40 $\mathrm{m}^{2}$. Un amplio acceso abierto localizado en el lado occidental de 5D20 permitió el ingreso a su interior, además de mirar hacia el patio o plaza principal del conjunto (ver Figura 3).

\subsection{Cisterna o chultún}

A esta unidad doméstica se asocia una cisterna o depósito de agua (chultún) que se encuentra a pocos metros al oeste de 5D19. El chultún fue construido en un afloramiento de roca madre que proporciona cierto declive hacia la boca; no se identificó ningún área de captación artificial. La construcción de este depósito de agua se realizó al haber excavado directamente en la roca madre, presenta una forma piriforme, la boca tiene un diámetro de $55 \mathrm{~cm}$, con una circunferencia bastante regular; el diámetro del cuello es de $60 \mathrm{~cm}$ en su parte más ancha y su largo es de $40 \mathrm{~cm}$. La profundidad máxima del Chultún 1 es de 1,75 metros y el diámetro interno máximo, localizado a un metro de profundidad desde la boca, es de 1,22 m.

Al considerar las dimensiones internas del chultún, podemos estimar que este depósito pudo haber almacenado hasta 475 litros de agua, una capacidad muy baja de almacenaje del vital líquido. Cabe notar que, si una persona consume entre dos y tres litros de agua al día, se estima que al año esta persona consumiría aproximadamente 912,5 litros de agua (2,5 litros diarios en promedio por 365 días). Por lo tanto, el chultún asociado con las estructuras mencionadas pudo haber almacenado agua para 
que fuera consumida hasta por cinco o seis individuos en menos de un mes. Tomando en cuenta estas estimaciones de consumo de agua en la unidad doméstica formada por 5D16, 5D19 y 5D20, resulta obvio que sus habitantes tuvieron acceso a otras fuentes de agua y debieron de haber almacenado este líquido de manera eficiente empleando otros medios o recursos. Por ejemplo, cabe la posibilidad que después del término de la temporada de lluvias, el chultún fuera rellenado de manera regular al haberse obtenido agua de otras fuentes localizadas en el mismo asentamiento, como pudo haber sido algún cenote.

\subsection{Banco de caliza}

Hacia la parte sureste del conjunto formado por las tres estructuras definidas, se halló un orificio en la roca madre y se determinó que de su interior se extrajo material de caliza para la construcción del conjunto arquitectónico. El que esta unidad doméstica haya contado con su propio banco de material de caliza no es extraño cuando consideramos los siguientes dos factores. Primero, la cantera de caliza está ubicada a muy corta distancia de las estructuras, por lo que esta cercanía debió de haber ayudado a reducir los costos de transportación de la caliza después de haber sido obtenida de la cantera. Segundo, el banco de material pudo haber proporcionado piedras de diferentes tamaños y polvo de construcción que se emplearon en la edificación de las estructuras (Hoil Chacón 2006; ver también Winemiller 1997).

\section{Materiales asociados a las estructuras investigadas}

\subsection{Metates}

Tres metates, o piedras de molienda, fueron hallados a un costado y al frente de la escalinata que conduce a la parte superior del basamento donde se eleva 5D16 (ver Figura 3; Figura 7). Estos metates son ápodos y presentan una zona o área cerrada en la cual se pudo haber realizado la molienda de maíz, sal y achiote (Pat Cruz 2006). Cuando se encuentran metates asociados con unidades domésticas, esto sugiere que fueron empleados para preparar alimentos o substancias requeridas en actividades efectuadas de manera cotidiana.

\subsection{Cerámica}

La cerámica asociada con 5D16 se encontró en dos basureros localizados a ambos lados de la escalinata que conduce al edificio. Las excavaciones en los cinco cuartos de 5D16 no revelaron concentraciones de materiales culturales, por lo que se supone que el interior del edificio fue conservado limpio durante su ocupación y antes de ser abandonado (Fernández 2008: 84).

Los materiales cerámicos hallados en 5D16 consistieron fundamentalmente en cazuelas Yokat Estriado y Piste Estriado, cazuelas Holactun y Xcanchakan, y platos y cazuelas Maxcanu. Además, estos materiales se encontraron asociados con cerámica Naranja Fino Silho y Balancan, Chablekal Gris y Tohil Plomizo (Jiménez Álvarez 2007: 141). Con la excepción de estas cuatro últimas, cuya elaboración y procedencia fue la cuenca baja del río Usumacinta (Naranja Fino Silho, Naranja Fino 


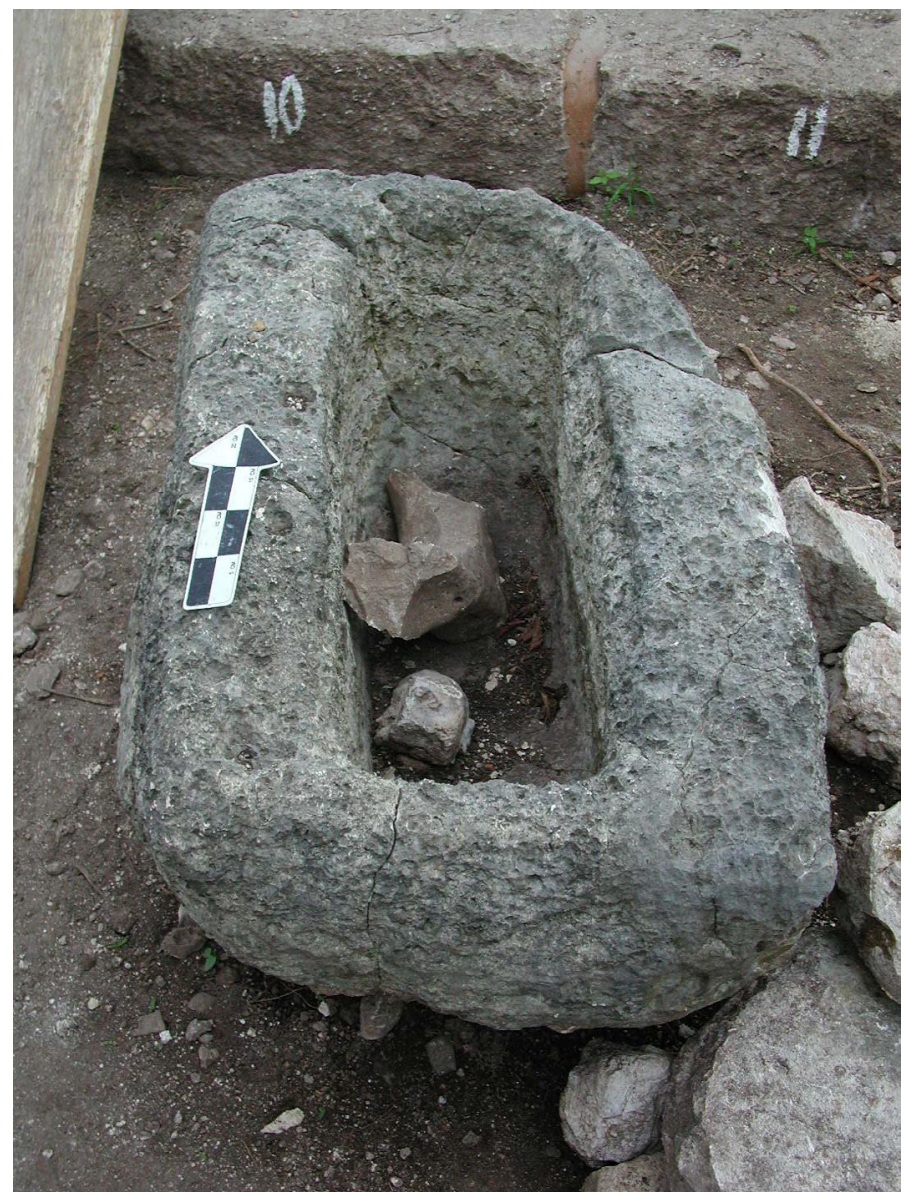

Figura 7. Metate ápodo de Sihó asociado a la Estructura 5D16 (fotografía de Rafael Cobos).

Balancan, Chablekal Gris) y las Tierras Altas del Occidente de Guatemala (Tohil Plomizo), el resto de los materiales cerámicos son originarios de la parte occidental y/o norte de Yucatán.

La cerámica recobrada de la Estructura 5D19 incluyó: ollas Yokat Estriado, ollas Maxcanu y Muna Engobe Blanco, ollas Sisal Sin Engobe, cazuelas Holactun y Muna Engobe Blanco, cuencos de los grupos cerámicos Ticul y Muna, platos trípodes Muna Engobe Blanco, ollas, cajetes y cuencos Naranja Fino Silho (Tun Ayora 2004: 140). De nueva cuenta, a excepción de los materiales Naranja Fino Silho que fueron manufacturados en la cuenca baja del río Usumacinta, los otros materiales cerámicos son propios del norte y oeste de Yucatán.

La cerámica reportada de la Estructura 5D20 incluyó: ollas Yokat Estriado, ollas Maxcanu y Muna Engobe Blanco, cerámica Naranja Fino Silho y Tohil Plomizo (Tun Ayora 2004:151). A excepción de los materiales Naranja Fino Silho y Tohil Plomizo originarios de la cuenca baja del río Usumacinta y Occidente de Tierras Altas de Guatemala, los otros materiales cerámicos fueron elaborados en el norte y oeste de Yucatán.

En resumen, las formas cerámicas identificadas en las estructuras 5D16, 5D19 y 5D20 incluyen cazuelas, platos, ollas, cajetes y cuencos. Estas formas sugieren no 
solamente que se prepararon alimentos en esas tres construcciones, sino también fueron consumidos - muy probablemente- en ellas. Además, la asociación de las diferentes formas cerámicas con los metates hallados en la unidad doméstica refuerza la idea de la preparación y el consumo de alimentos por parte de los residentes de 5D16, 5D19 y 5D20.

\subsection{Sílex}

En este trabajo reportamos un total de 2.163 artefactos de sílex hallados en 5D16 que incluyen: lascas de adelgazar $(\mathrm{n}=724)$, navajas prismáticas $(\mathrm{n}=289)$, bifaciales $(\mathrm{n}=53)$, lascas de descortezar $(n=35)$, lascas casuales $(n=19)$, lascas con retoque bifacial $(=7)$, núcleos de lasca casual $(\mathrm{n}=6)$, núcleo de navaja prismática $(\mathrm{n}=1)$, pedazos $(\mathrm{n}=450)$, lascas no asignadas a ninguna industria ( $\mathrm{n}=579$; ver Fernández 2008: 84-85).

En la Estructura 5D19 se encontraron bifaciales pequeños $(\mathrm{n}=2)$, bifaciales de punta lanceolada $(n=2)$, lascas $(n=43)$, macrolascas $(n=1)$, navajas prismáticas $(\mathrm{n}=61)$, núcleos de lasca casual $(\mathrm{n}=2)$, nódulo usado como alisador $(\mathrm{n}=1)$, pedazos $(n=24)$. Por otro lado, de la estructura 5D20 identificamos bifaciales $(n=7)$, lascas $(n=33)$, navajas prismáticas $(n=44)$, núcleos $(n=6)$, pedazos $(n=29)$.

El análisis de las herramientas de sílex halladas sugiere que en estas tres estructuras se estaban elaborando y re-afilando herramientas líticas. Además, el sílex hallado en Sihó puede ser originario de canteras ubicadas en el sur de Yucatán y/o la región de Río Bec en el sur de Campeche.

\subsection{Obsidiana}

Los artefactos de obsidiana recuperados e identificados en 5D16 incluyen: navajas prismáticas $(n=190)$, lascas (n 9), fragmentos de lascas $(n=33)$, fragmentos de núcleos poliédricos $(n=4)$, pedazos $(n=51)$, perforadores $(n=2)$, fragmento de bifacial $(\mathrm{n}=1)$, pieza no identificada $(\mathrm{n}=1)$, hasta un total de 315 artefactos; mientras que en $5 \mathrm{D} 19$ se reportaron un total de 33 piezas distribuidas en navajas prismáticas $(\mathrm{n}=31)$, punta de navaja $(\mathrm{n}=1)$ y perforador $(\mathrm{n}=1)$; apenas se recobraron 5 piezas en $5 \mathrm{D} 20$, entre navajas prismáticas $(\mathrm{n}=4)$ y una punta de navaja $(\mathrm{n}=1)$ (Tun Ayora 2004: 140, 155; Fernández 2008: 91).

Las fuentes de obsidiana identificadas de las que proceden los artefactos de la unidad doméstica son El Chayal, Ixtepeque y San Martín Jilotepeque en Guatemala; Ucareo/Zaragoza, Pachuca, Pico de Orizaba y Paredón en el centro y occidente de México. Además, los objetos de obsidiana sugieren elaboración y re-afilamiento de herramientas líticas.

\subsection{Panel 1}

Durante los trabajos de investigación de la Estructura 5D16 se encontró el Panel 1 (Figura 8). En el momento de su hallazgo el monumento se encontraba caído, con la cara esculpida hacia abajo, a unos dos metros del talud situado al oeste de la escalinata sur del edificio (ver Figura 3) (Fernández y Vázquez de Ágredos 2002: 62). El paramento del talud aún presentaba evidencias del espacio donde estuvo empotrado originalmente el monumento, por lo que se pudo determinar su ubicación exacta respecto a la estructura. 

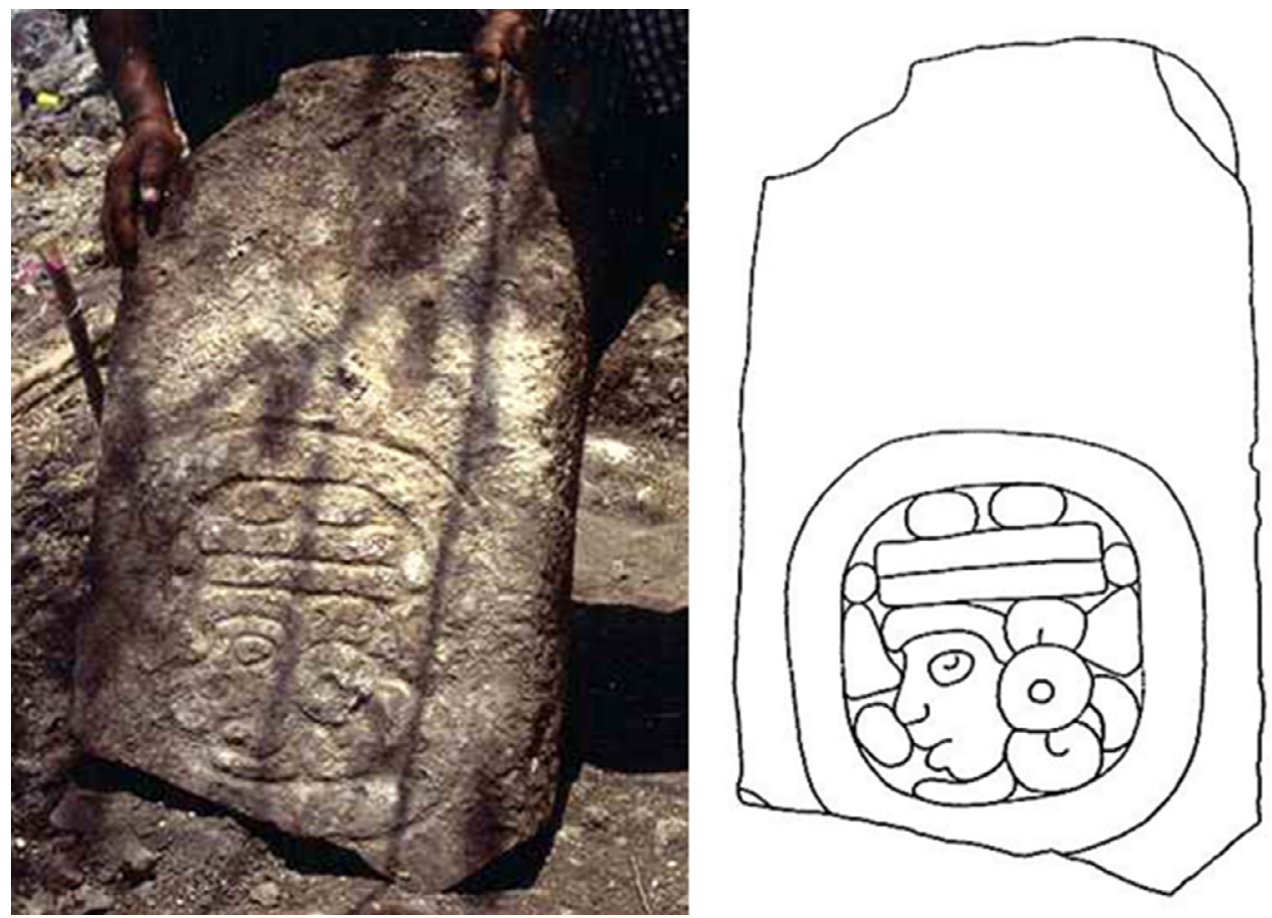

Figura 8. Panel 1 de Sihó (fotografía de Rafael Cobos, dibujo de Alfonso Lacadena García-Gallo).

La fisonomía del monumento y su clara asociación arquitectónica lo señalan como un panel, siendo el primero de estas características en ser documentado en el sitio. Las medidas del panel son: $0,75 \mathrm{~m}$ de alto por 0,50 $\mathrm{m}$ de largo. Dado que el monumento está fracturado en su parte inferior, la altura original de la pieza sería mayor. El monumento presenta una inscripción esculpida en bajorrelieve, de $0,3 \mathrm{~cm}$ de profundidad.

El estado de conservación del monumento y su relieve es aceptable, aunque el monumento se encuentra fracturado transversalmente en su parte inferior, habiendo afectado levemente la línea de fractura a la parte inferior del contorno del cartucho circular donde se inserta el texto glífico. En sus esquinas superiores se aprecian otras fracturas leves.

La inscripción consta de un único bloque glífico inscrito en un cartucho circular (Figura 8). El bloque consta de tres signos: un numeral 12 expresado en el sistema de puntos y barras, un logograma Ajaw, escrito en variante de cabeza, un signo silábico wa ubicado como postfijo del logograma, actuando de complemento fonético.

\section{2-AJAW-wa \\ 12 Ajaw \\ «12 Ajaw»}

El texto consiste en una breve anotación calendárica. Con toda seguridad se trata de una fecha en Cuenta Corta señalando un final de $k$ 'atún, sistema de datación favorecido en el norte de las Tierras Bajas mayas. Las cuentas largas a las que podría corresponder un k'atún 12 Ajaw dentro del periodo Clásico maya son las siguientes: 
12 Ajaw 8 Sotz' (7 de julio de 396 d.C.)

12 Ajaw 8 Keh (11 de octubre de 652 d.C.)

12 Ajaw 3 Wo (15 de enero de 909 d.C. $)^{3}$

De estas tres fechas, la primera y última podrían descartarse por criterios estilísticos, por lo que se favorece la fecha central de 9.11.0.0.0 12 Ajaw 8 Keh (11 de octubre de 652 d.C.) como la más probable. Dada la ubicación del Panel 1 en el paramento de uno de los taludes del basamento de la Estructura 5D16, y la funcionalidad que este tipo de fechas asociadas a soportes arquitectónicos suele tener, se puede tomar la fecha de 652 d.C. - o cualquier otra perteneciente al periodo 633-652 d.C. que comprende el k'atún- como la fecha de construcción de la estructura.

El Panel 1 ofrece la particularidad de presentar el texto inscrito en un cartucho circular. Inscripciones presentado este formato -bien simple, con un solo bloque glífico, o complejo, con más de un bloque, bien con cartucho circular delineado o rebajado- se documentan durante todo el periodo Clásico en otros sitios del Área Maya, como en Tikal (Hombre de Tikal, ca. 8.18.10.8.12, paneles de estuco), Uaxactún (Estela 6, 9.6.0.0.0; Estela 17); Dzibanché (Dintel 3, 9.6.0.0.0), El Resbalón (escaleras jeroglíficas 1, 2 y 3, ca. 9.7.6.4.18), Caracol (Estela 5, 9.9.0.4.0, Estela 6, 9.8.10.0.0, Estela 19, 9.19.10.0.0, Estela 20), Naranjo (Escalera Jeroglífica 1, 9.10.10.0.0), Tortuguero (Estela 1, 9.11.0.0.0, Monumento 8, ca. 9.12.6.17.18), Palenque (Templo de las Inscripciones, paneles de estuco, $c a .9 .12 .11 .12 .10)$, La Lagunita (Estela 4), Etzná (Escalera Jeroglífica ca. 9.10.10.0.0), Oxkintok (Estructura 3C10), Xburrotunich (Jamba 1), Sitio Ch'ich', San Francisco (Estela 2, Estela 3) y Sayil (Estela 3, 10.3.0.0.0?). Si atendemos a las fechas probables de dedicación de los monumentos, podemos ver que es un formato de escritura no infrecuente a finales del Clásico Temprano y comienzos del Clásico Tardío.

Ese formato de escritura apareció sobre todo en el extremo norte de la zona Puuc y área aledaña durante el Clásico Tardío, tanto en soportes monumentales -los ya mencionados de Oxkintok, San Francisco, Xburrotunich, Sitio Ch'ich' y ahora Sihócomo cerámicos según atestiguan los ejemplos en alfarería Chocholá. Algunos investigadores han sugerido que esa zona geográfica pudo haber sido uno de los principales focos de producción de la cerámica Chocholá (Coe 1973; Tate 1985; Grube 1990; García Campillo 1992; Ardren 1996; Werness 2010).

\section{Conclusiones}

La evidencia material hallada en una unidad doméstica de Sihó formada por las estructuras 5D16, 5D19 y 5D20 proporciona datos para responder a tres preguntas planteadas líneas arriba. Estas preguntas están relacionadas con el tipo de actividades realizadas en esas tres estructuras, quiénes fueron las personas que residieron en el conjunto, y cuál pudo haber sido su relación con miembros de la elite que ocuparon el centro del sitio ubicado a $200 \mathrm{~m}$ al sureste (ver Figura 2).

En referencia a las actividades que realizaron de manera cotidiana en la unidad doméstica, reconocemos por lo menos seis de ellas. Primera actividad, los metates $\mathrm{y}$ las diferentes formas cerámicas sugieren elaboración -en parte por molienda- y

3 Las fechas cristianas están expresadas en calendario Juliano. 
consumo de alimentos utilizando ollas, platos, cazuelas, cuencos y cajetes. Segunda actividad, mantenimiento por medio de re-afilamiento de herramientas líticas hechas de obsidiana y sílex que pudieron haberse utilizado para cortar/destazar y/o rallar carne, piel y otros productos animales, así como vegetales y minerales. Tercera actividad, la cisterna asociada a la unidad doméstica sugiere consumo de agua tanto por los residentes del conjunto como -posiblemente- por sus animales domésticos (¿venados?). Cuarta actividad, uso de espacios abiertos -como la pequeña plaza central- para la constante interacción entre ocupantes permanentes del conjunto, así como de visitantes. Quinta actividad, recordar de manera constante -al mirar el Panel 1- la fecha de fundación y/o construcción del conjunto arquitectónico relacionada - quizás- con ancestros comunes. Sexta actividad, acceso a, o abastecimiento de, materiales cerámicos, de obsidiana y sílex de origen foráneo que pudieron haber sido considerados como objetos suntuarios como parte de la alta posición social que gozaban los residentes de 5D16, 5D19 y 5D20.

En relación a los residentes permanentes de la elaborada unidad doméstica, la evidencia material sugiere que se trató de individuos miembros de la elite quienes ocuparon la Estructura 5D16. Aparentemente, estos individuos tuvieron acceso y -posiblemente- control de fuerza de trabajo para efectuar labores arquitectónicas que incluyeron la construcción y/o mantenimiento de las tres estructuras. Además, algunos miembros de elite pudieron haber sido hábiles escribanos para realizar la inscripción de la fecha 12 Ajaw en el Panel 1, o bien, emplearon los servicios de algún escribano de la comunidad para realizar la inscripción que -aparentementeconmemora un evento histórico.

Las estructuras 5D19 y 5D20 formaron parte fundamental de la unidad doméstica al compartir la plaza o patio central del conjunto. Sin embargo, estas dos se ubican en un plano más bajo y, considerando su diseño constructivo más simple que el de 5D16, podemos sugerir que ambas estructuras pudieron haber albergado a individuos que actuaron como sirvientes para realizar las actividades cotidianas que demandaban los miembros de la elite que residían en 5D16.

En cuanto a la pregunta sobre la relación que pudieron haber tenido los residentes de esta unidad doméstica con miembros de la elite quienes vivían en la parte central de Sihó, podemos argumentar lo siguiente. Primero, existió una estrecha relación entre miembros de la elite de la parte central y aquellos de la unidad doméstica ya que estaban emparentados por lazos consanguíneos. Por lo tanto, la evidencia material hallada en este conjunto residencial revela una estrecha relación familiar en la cual los residentes de estas tres estructuras fueron beneficiados - por ejemplo- con regalos elaborados en cerámica, obsidiana y sílex. Esta acción de recibir formó parte de una reciprocidad generalizada entre miembros de la elite relacionados por lazos de sangre.

Segundo, los miembros de la elite residentes de la unidad doméstica en cuestión no necesariamente estaban emparentados por lazos consanguíneos con aquellos individuos residiendo en el centro de Sihó. Podemos suponer que el acceso a bienes suntuarios que tuvieron los residentes del grupo habitacional investigado pudo haberse debido al intercambio, tanto por redistribución centralizada como por reciprocidad, realizado por otros miembros de la elite de Sihó. Si este fue el caso, los ocupantes de 5D16, 5D19 y 5D20 recibieron vasijas cerámicas, herramientas de obsidiana y sílex como bienes suntuarios $\mathrm{y}$, en intercambio, pudieron haber brindado fuerza de 
trabajo y/o productos agrícolas para corresponder con otros miembros privilegiados, poderosos y con opulencia que habitaron Sihó durante el periodo Clásico Tardío.

En conclusión, el estudio realizado sobre una unidad doméstica de Sihó revela el enorme potencial de interpretación que, en este caso, los datos arqueológicos y epigráficos permiten para un pequeño universo dentro de una comunidad maya. Además, nuestro análisis contribuye a caracterizar arqueológicamente el «Asentamiento Tipo B» que Willey (1956) definió hace más de seis décadas y del cual hoy día se reportan numerosos ejemplos en las Tierras Bajas mayas. Por último, nuestro análisis de la unidad doméstica de Sihó formada por las estructuras 5D16, 5D19 y 5D20 se suma a las interpretaciones que existen en la actualidad para explicar o caracterizar un tipo peculiar de asentamiento. En otras palabras, los «Asentamientos tipo B» reflejan la forma en la cual los miembros de un conjunto social dispusieron y organizaron su comunidad en el occidente de Yucatán.

\section{Referencias}

Ardren, Traci. 1996. «The Chocholá Ceramic Style of Northern Yucatan: An Iconographic and Archaeological Study», en Eighth Palenque Round Table, 1993, Martha J. Macri y Jan McHargue, eds., pp. 237-245. San Francisco: Pre-Columbian Art Research Institute.

Barnhart, Edwin L. 2006. «Indicators of Urbanism at Palenque», en Palenque, Recent Investigations at the Classic Maya Center, Damien B. Marken, ed., pp. 107-121. Maryland: Altamira Press.

Chase, Arlen F. 1998. «Planeación cívica e integración de sitio en Caracol, Belice: definiendo una economía administrada del periodo Clásico Maya», en Los Investigadores de la Cultura Maya 6, pp. 27-44. Campeche: Universidad Autónoma de Campeche.

Chase, Diane Z. y Arlen F. Chase. 1992. «Mesoamerican Elites: Assumptions, Definitions, and Models», en Mesoamerican Elites. An Archaeological Assessment, Diane Z. Chase y Arlen F. Chase, eds., pp. 3-17. Norman: University of Oklahoma Press.

Cobos, Rafael. 2003. "Ancient Community Form and Social Complexity at Chichén Itzá», en Urbanism in Mesoamerica, Vol. I, William.T. Sanders, Alba G. Mastache y Robert H. Cobean, eds., pp. 451-472. México y Pennsylvania: Instituto Nacional de Antropología e Historia y The Pennsylvania State University.

Coe, Michael. 1973. The Maya Scribe and His World. Nueva York: The Grolier Club.

Doxiadis, Constantinos A. 1968. Ekistics: An Introduction to the Science of Human Settlement. Londres: Oxford University Press.

Fash, William L. 1983. «Deducing Social Organization from Classic Maya Settlement Patterns: A Case Study from the Copan Valley», en Civilization in the Ancient Americas, Essays in Honor of Gordon R. Willey, Richard M. Leventhal y Alan L. Kolata, eds., pp. 261-288. Albuquerque y Cambridge: University of New Mexico Press, Peabody Museum of Archaeology and Ethnology, Harvard University.

Fernández Souza, Lilia. 2008. Grupos domésticos y espacios habitacionales en las Tierras Bajas Mayas durante el periodo Clásico. Tesis doctoral. Hamburgo: Universidad de Hamburgo.

Fernández Souza, Lilia y María Luisa Vázquez de Ágredos. 2002. «Informe preliminar de la excavación horizontal realizada en la Estructura 5D16 de Sihó», en Proyecto Arqueológico El Surgimiento de la Civilización en el Occidente de Yucatán: Los Orígenes de la Complejidad Social en Sihó, Rafael Cobos, Lilia Fernández, Vera Tiesler, Pilar Zabala, 
Armando Inurreta, Nancy Peniche, Ma Luisa Vázquez de Ágredos y Diana Pozuelo, eds., pp. 48-68. México: Informe de actividades de la Temporada de Campo 2001 presentado al Consejo de Arqueología del Instituto Nacional de Antropología e Historia.

Fernández Souza, Lilia, Rafael Cobos y María Luisa Vázquez de Ágredos. 2003a. «Análisis de una estructura tipo palacio en Sihó, Yucatán», en XVI Simposio de Investigaciones Arqueológicas en Guatemala 2002, Juan Pedro Laporte, Bárbara Arroyo, Héctor L. Escobedo y Héctor E. Mejía, eds., pp. 1031-1036. Guatemala: Museo Nacional de Arqueología y Etnología.

_. 2003b «Arquitectura en Sihó, Yucatán: la Estructura 5D16», en Los Investigadores de la Cultura Maya 11, pp. 354-361. Campeche: Universidad Autónoma de Campeche.

Fernández Souza, Lilia, Rafael Cobos, Socorro Jiménez Álvarez, Christopher Götz, Vera Tiesler Blos. 2010. «Morir al filo del tiempo: un entierro infantil colonial en urna en Sihó, Yucatán». Mexicon XXXII (4): 82-87.

Folan, William J., Laraine A. Fletcher, Jacinto May Hau, y Linda Florey Folan. 2001. Las ruinas de Calakmul, Campeche, México. Un lugar central y su paisaje cultural. Campeche: Centro de Investigaciones Históricas y Sociales, Universidad Autónoma de Campeche.

Folan, William J., Ellen R. Kintz y Laraine A. Fletcher. 1983. Coba: A Classic Maya Metropolis. Nueva York: Academic Press.

Gallareta Negrón, Tomás. 1984. Cobá. Forma y función de una comunidad maya prehispánica. Tesis de Licenciatura, Facultad de Ciencias Antropológicas. Mérida: Universidad Autónoma de Yucatán.

García Campillo, José Miguel. 1992. «Informe epigráfico sobre Oxkintok y la cerámica Chocholá». Oxkintok 4, pp. 185-200. Madrid: Misión Arqueológica de España en México.

Garza Tarazona, Silvia y Edward B. Kurjack. 1980. Atlas Arqueológico del Estado de Yucatán. México: Instituto Nacional de Antropología e Historia.

Graham, Elizabeth. 1999. «Stone Cities, Green Cities», en Complex Polities in the Ancient Tropical World, Elisabeth A. Bacus y Lisa J. Lucero, eds., pp. 185-194. Arlington: American Anthropological Association.

Grube, Nikolai. 1990. «The Primary Standard Sequence on Chocholá Style Ceramics», en The Maya Vase Book 2, Justine Kerr, ed., pp. 320-330. Nueva York: Kerr Associates.

Harrison, Peter D. y Billie L. Turner II. 1978. Pre-Hispanic Maya Agriculture. Albuquerque: University of New Mexico Press.

Haviland, William A. 1965. «Prehistoric Settlement at Tikal, Guatemala». Expedition 7: 1423.

Haviland, William A. y Hattula Moholy-Nagy. 1992. «Distinguishing the High and Mighty from the Hoi Polloi at Tikal, Guatemala», en Mesoamerican Elites. An Archaeological Assessment, Diane Z. Chase y Arlen F. Chase, eds., pp. 50-79. Norman: University of Oklahoma Press.

Hoil Chacón, Santos W. 2006. Un punto de vista del cálculo arquitectónico: la Estructura 5 D16 de Sihó, Yucatán. Tesis de Licenciatura, Facultad de Ciencias Antropológicas. Mérida: Universidad Autónoma de Yucatán.

Jiménez Álvarez, Socorro. 2007. Sihó: una unidad política del Occidente de Yucatán. Tesis de Maestría, Facultad de Ciencias Antropológicas. Mérida: Universidad Autónoma de Yucatán.

Kurjack, Edward B. 1974. Prehistoric Lowland Maya Community and Social Organization. A Case Study at Dzibilchaltun, Yucatan, Mexico. Middle American Research Institute 38. Nueva Orleans: Tulane University. 
. 1994. «Political Geography of the Yucatecan Hill Country», en Hidden among the Hills. Maya Archaeology of the Northwest Yucatan Peninsula, Hans J. Prem, ed., pp. 308-315. Acta Mesoamericana 7. Möckmühl: Verlag von Flemming.

Kurjack, Edward B. y Silvia Garza Tarazona. 1981. «PreColumbian Community Form and Distribution in the Northern Maya Area», en Lowland Maya Settlement Patterns, Wendy Ashmore, ed., pp. 287-309. Albuquerque: University of New Mexico Press.

Landa, Diego de. 1959. Relación de las cosas de Yucatán. México: Editorial Porrúa.

Pat Cruz, Edgar Daniel. 2006. Análisis de las piedras de molienda de Sihó, Yucatán. Tesis de Licenciatura, Facultad de Ciencias Antropológicas. Mérida: Universidad Autónoma de Yucatán.

Tate, Carolyn. 1985. «The Carved Ceramics Called Chocholá», en Fifth Palenque Round Table, 1983, Merle Greene Robertson y Virginia M. Fields, eds., pp. 123-129. San Francisco: The Pre-Columbian Art Research Institute.

Tourtellot, Gair. 1988. Excavations at Seibal, Department of Peten, Guatemala: Peripheral Survey and Excavation Settlement and Community Patterns. Memoirs of the Peabody Museum 16. Cambridge: Harvard University.

Tourtellot, Gair, Jeremy A. Sabloff y Kelli Carmean. 1992. «'Will the Real Elites Please Stand Up?': An Archaeological Assessment of Maya Elite Behavior in the Terminal Classic Period», en Mesoamerican Elites. An Archaeological Assessment, Diane Z. Chase y Arlene F. Chase, eds., pp. 80-98. Norman: University of Oklahoma Press.

Tun Ayora, Gabriel E. 2004. La organización de viviendas mayas prehispánicas: análisis de estructuras domésticas asociadas a unidades habitacionales de elite de Sihó, Yucatán. Tesis de Licenciatura, Facultad de Ciencias Antropológicas. Mérida: Universidad Autónoma de Yucatán.

Werness, Maline D. 2010. Chocholá Ceramics and the Polities of Northwestern Yucatán. Tesis doctoral. Faculty of the Graduate School. Austin: The University of Texas at Austin.

Willey, Gordon R. 1956. «Problems Concerning Prehistoric Settlement Patterns in the Maya Lowlands», en Prehistoric Settlement Patterns in the New World, Gordon R. Willey, ed., pp. 107-114. Nueva York: Wenner-Gren Foundation.

Winemiller, Terance L. 1997. Exploitation of Limestone Resources by the Ancient Maya at Chichen Itza, Yucatan, Mexico. Tesis de Maestría, Department of Geography and Anthropology. Baton Rouge: Louisiana State University. 